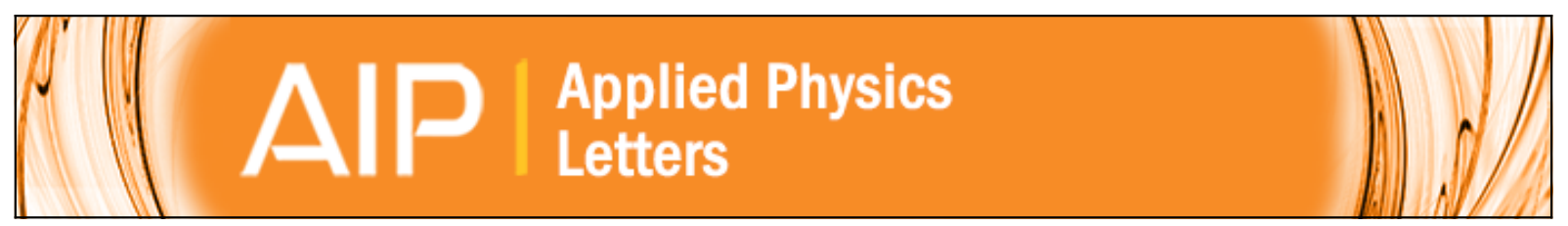

\title{
Pentacene thin films on ferromagnetic oxide: Growth mechanism and spintronic devices
}

P. Graziosi, A. Riminucci, M. Prezioso, C. Newby, D. Brunel, I. Bergenti, D. Pullini, D. Busquets-Mataix, M. Ghidini, and V. A. Dediu

Citation: Applied Physics Letters 105, 022401 (2014); doi: 10.1063/1.4890328

View online: http://dx.doi.org/10.1063/1.4890328

View Table of Contents: http://scitation.aip.org/content/aip/journal/apl/105/2?ver=pdfcov

Published by the AIP Publishing

\section{Articles you may be interested in}

Hanle effect missing in a prototypical organic spintronic device

Appl. Phys. Lett. 102, 092407 (2013); 10.1063/1.4794408

Manipulating spin injection into organic materials through interface engineering

Appl. Phys. Lett. 101, 022416 (2012); 10.1063/1.4737008

Planar-type spin valves based on low-molecular-weight organic materials with $\mathrm{La} 0.67 \mathrm{Sr} 0.33 \mathrm{Mn} \mathrm{O} 3$ electrodes Appl. Phys. Lett. 92, 153304 (2008); 10.1063/1.2905288

Room temperature organic spintronics

J. Appl. Phys. 103, 07F306 (2008); 10.1063/1.2829245

Application of regioregular polythiophene in spintronic devices: Effect of interface

Appl. Phys. Lett. 89, 122114 (2006); 10.1063/1.2356463

\section{A|P| $\left.\right|_{\text {Applied Physics }} ^{\text {Journal of }}$}

Journal of Applied Physics is pleased to announce André Anders as its new Editor-in-Chief 


\title{
Pentacene thin films on ferromagnetic oxide: Growth mechanism and spintronic devices
}

\author{
P. Graziosi, ${ }^{1,2}$ A. Riminucci, ${ }^{1}$ M. Prezioso, ${ }^{1, \text { a) }}$ C. Newby, ${ }^{1, b)}$ D. Brunel, ${ }^{1, c)}$ I. Bergenti, ${ }^{1}$ \\ D. Pullini, ${ }^{3}$ D. Busquets-Mataix,,$^{2,4}$ M. Ghidini, ${ }^{5,6}$ and V. A. Dediu ${ }^{1}$ \\ ${ }^{1}$ CNR - ISMN, Consiglio Nazionale delle Ricerche - Istituto per lo Studio dei Materiali Nanostrutturati, \\ v. Gobetti 101, 40129 Bologna, Italy \\ ${ }^{2}$ Instituto de Tecnología de Materiales, Universitat Politécnica de Valencia, Camino de Vera s/n, 46022, \\ Valencia, Spain \\ ${ }^{3}$ Centro Ricerche Fiat, 10043 Orbassano (TO), Italy \\ ${ }^{4}$ Departamento de Ingeniería Mecánica y de Materiales, Universitat Politécnica de Valencia, \\ Camino de Vera s/n, 46022, Valencia, Spain \\ ${ }^{5}$ Department of Physics, University of Parma, v. le G.P. Usberti 7/A, 43100 Parma, Italy \\ ${ }^{6}$ Department of Materials Science, University of Cambridge, Cambridge CB3 OFS, United Kingdom
}

(Received 27 May 2014; accepted 2 July 2014; published online 14 July 2014)

\begin{abstract}
We investigated the growth mechanism of pentacene thin films on $\mathrm{La}_{0.7} \mathrm{Sr}_{0.3} \mathrm{MnO}_{3}$. A diffusion limited, thermally activated growth was found. Pentacene molecules formed flat islands that were a few microns in size and whose growth during deposition showed a strong anisotropy. We extracted a nucleation energy of $0.65 \pm 0.05 \mathrm{eV}$ and a diffusion barrier energy of $0.7 \pm 0.2 \mathrm{eV}$. We also estimated a critical nucleus size of three molecules. We show that vertical pentacene-based spintronic devices with $\mathrm{La}_{0.7} \mathrm{Sr}_{0.3} \mathrm{MnO}_{3}$ and Co electrodes demonstrated magnetoresistive effects up to room temperature. We also propose a route for pentacene-based spintronic devices improvement. ( 2014 AIP Publishing LLC. [http://dx.doi.org/10.1063/1.4890328]
\end{abstract}

Spintronics is a branch of electronics, where the information is carried by the spin degree of freedom of the electron. Thanks to their small spin-orbit coupling, ${ }^{1}$ organic semiconductors (OSCs) are promising candidates as spin transporting media in spintronics. ${ }^{2}$ Since the first successful demonstration of spin transport through organic semiconductors, ${ }^{3}$ organic spintronics have been the object of increasing interest and gave rise to conceptually new devices. ${ }^{4,5}$

Electrically driven spintronic devices are usually fabricated by separating two spin polarized electrodes by a charge and spin conducting non-magnetic layer. The detected effect consists in a resistance modification caused by the variation of the relative spin polarization of the electrodes. The parallel and the antiparallel configurations have two distinct resistances, resulting in the so called spin valve effect.

Aromatic hydrocarbon semiconductors have the best electrical properties among OSCs. ${ }^{6}$ In particularly, pentacene, which has the relatively high mobility of $5 \mathrm{~cm}^{2} /(\mathrm{Vs}),{ }^{7}$ is one of the most used in organic electronics. ${ }^{6}$ Despite this, its investigation in spintronics is limited to a very few cases. Spintronic effects at $5.3 \mathrm{~K}$ were reported for planar devices with $\mathrm{La}_{0.7} \mathrm{Sr}_{0.3} \mathrm{MnO}_{3}$ (LSMO) electrodes and a pentacene spacer $^{8}$ (a spin diffusion length exceeding $200 \mathrm{~nm}$ was proposed in this paper) and in vertical devices comprising a $\mathrm{Fe}$ top electrode and a composite $\mathrm{Co}: \mathrm{TiO}_{2}$ bottom electrode separated by a $100 \mathrm{~nm}$ thick pentacene spacer. ${ }^{9}$ On the other hand, no magnetoresistance (MR) effects were detected in pentacene-based devices fabricated without oxide

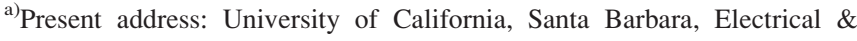
Computer Engineering Harold Frank Hall, Santa Barbara, CA 93106-9560.

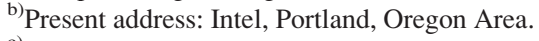

${ }^{c}$ Present address: Ecole Polytechnique, Route de Saclay, Palaiseau, France.
}

electrodes,${ }^{10}$ unless oxidizing the pentacene layer in plasma oxygen. ${ }^{11}$ Thus, available data point to the crucial role of oxides, and in particular of LSMO, as electrode materials for pentacene-based spintronics; indeed, LSMO is by far the most used spin polarized electrode in organic spintronics. ${ }^{12}$

In this report, we present a spin valve device based on pentacene as OSC spacer and on LSMO and Co as ferromagnetic electrodes, operating up to room temperature (RT). In order to pave the way for improved spintronic devices, we also investigated the pentacene growth on top of LSMO thin film surfaces.

LSMO films were grown on $\mathrm{SrTiO}_{3}(100)$ (STO) with a homemade channel spark ablation set-up. ${ }^{13}$ Prior to pentacene deposition, LSMO films were annealed in situ in ultra high vacuum (UHV) in order to clean the surface and to restore the surface properties. ${ }^{14}$ Pentacene films with thicknesses between $3 \mathrm{~nm}$ and $100 \mathrm{~nm}$ were subsequently deposited at a base pressure of $10^{-8}$ mbar with a homemade organic molecular beam deposition system at a fixed rate of $0.003 \mathrm{~nm} / \mathrm{s}$. Atomic force microscopy (AFM) was carried out with a Nanoscope III microscope, and the analysis was performed with the Gwyddion software package. ${ }^{15}$

Figure 1 presents the resistance of a LSMO/pentacene/ $\mathrm{Al}_{2} \mathrm{O}_{3} / \mathrm{Co}$ spin valve as function of the in plane applied magnetic field $\mathbf{H}$, the pentacene thickness is 100 and $300 \mathrm{~nm}$; area junction, defined by shadow masking, is $1 \mathrm{~mm}^{2}$. The measurement was carried out with a Keithley 236 in the four probe configuration, with an applied bias of $-0.1 \mathrm{~V}$ (grounded $\mathrm{Co}$ ) at $100 \mathrm{~K}$ and at room temperature. Similar structures, with $\mathrm{Alq}_{3}$ as OSC spacer, were widely studied. ${ }^{4,12,16,17}$ The Al oxide buffer layer was deposited by channel spark ablation of an alumina target in oxygen environment at room temperature ${ }^{18}$ in order to preserve the 


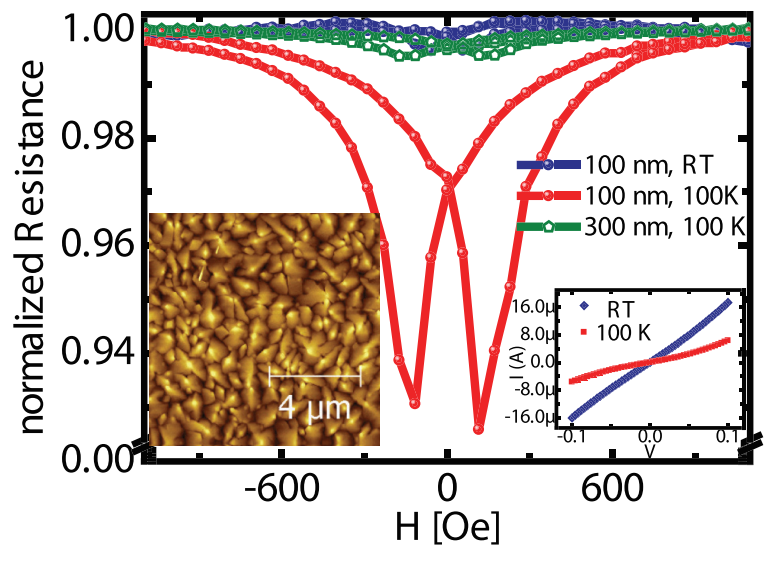

FIG. 1. Magnetoresistance of a LSMO/pentacene $(100 \mathrm{~nm}$ and $300 \mathrm{~nm}) /$ $\mathrm{Al}_{2} \mathrm{O}_{3}(2.5 \mathrm{~nm}) / \mathrm{Co}$ spin valve measured at $100 \mathrm{~K}$ and at $\mathrm{RT}$ with a $-0.1 \mathrm{~V}$ applied bias (grounded Co). The left hand side inset shows a $10 \times 10 \mu \mathrm{m}^{2}$ AFM image of the pentacene film covered by $2 \mathrm{~nm}$ of $\mathrm{Al}_{2} \mathrm{O}_{3}$; the $\mathrm{z}$ scale is $45 \mathrm{~nm}$. The right hand side inset shows the IV curves at room temperature and $100 \mathrm{~K}$ for the $100 \mathrm{~nm}$ case; their behaviour with temperature is semiconducting-like.

properties of both pentacene and $\mathrm{Co}$, as done in $\mathrm{Alq}_{3}$ based devices. ${ }^{12,18}$ After each step, the vacuum was broken for a couple of minutes to change the shadow masks. The pentacene films were deposited at room temperature in order to make the results comparable to those from the previous report on LSMO/pentacene spin valves ${ }^{8}$ and to those obtained with other OSCs. ${ }^{4,8,10,17,19}$ An AFM micrograph of the pentacene surface after the $\mathrm{Al}_{2} \mathrm{O}_{3}$ deposition is reported in the left inset in Figure 1.

The figure of merit of the spin valve device is its $\mathrm{MR}=\left(\mathrm{R}_{\mathrm{AP}}-\mathrm{R}_{\mathrm{P}}\right) / \mathrm{R}_{\mathrm{P}}$, where $\mathrm{R}_{\mathrm{P}}$ and $\mathrm{R}_{\mathrm{AP}}$ are the device resistance in the parallel and antiparallel state, respectively. The device with $100 \mathrm{~nm}$ nominal pentacene layer shows a MR of $-7 \%$ at $100 \mathrm{~K}$ and $-0.4 \%$ at $\mathrm{RT}$, which is in line with the room temperature $\mathrm{MR}$ of $\mathrm{Alq}_{3}$-based spin valves. ${ }^{16}$ In analogy with $\mathrm{Alq}_{3}$ devices, the negative sign of the MR can be tentatively attributed to the interface energy levels alignment. ${ }^{16,20}$ Since the nominal thickness of the pentacene film is $100 \mathrm{~nm}$ and the peak to valley height difference is $45 \mathrm{~nm}$, conductive paths of different lengths are present, and hence, the effective spin transport length is much smaller than the nominal thickness of the pentacene spacer. Nevertheless, the Co and LSMO electrodes are not shorted: the current-voltage characteristic (right inset in Figure 1) as a function of temperature shows a semiconducting behaviour. The device with $300 \mathrm{~nm}$ pentacene nominal thickness shows, at $100 \mathrm{~K}$, a MR about $-0.3 \%$ and no MR at RT, a similar trend was observed in devices based on transport in $\mathrm{Alq}_{3} \cdot{ }^{16}$ Considering also that the sign of the MR of LSMO/ $\mathrm{Al}_{2} \mathrm{O}_{3} / \mathrm{Co}$ junctions is the opposite of the one we detect, ${ }^{21}$ we rule out pinholes effects.

Having demonstrated the suitability of pentacene as a spin transporting medium in combination with LSMO, it is necessary to study the pentacene film growth mechanism on LSMO in order to establish the relevant parameters for further device improvement.

The LSMO surface (Figure 2(a)) showed $200 \mathrm{~nm}$ wide terraces with a roughness smaller than a unit cell: the root mean square roughness $R_{r m s}$ was about $0.13 \pm 0.02 \mathrm{~nm}$.

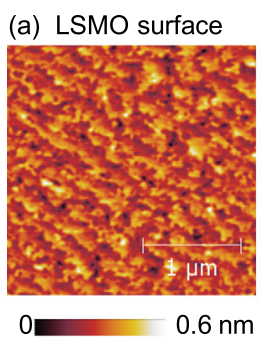

(d) $20 \mathrm{~nm} @ 0{ }^{\circ} \mathrm{C}$

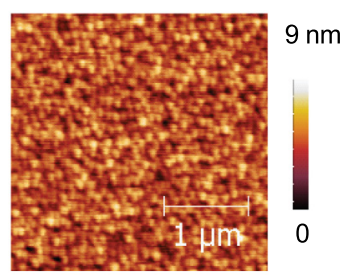

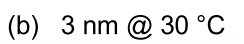

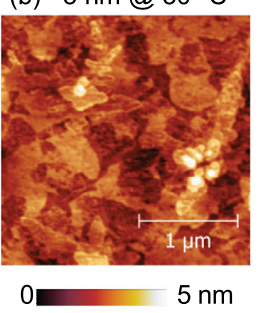

(c) $100 \mathrm{~nm} @ 30^{\circ} \mathrm{C}$

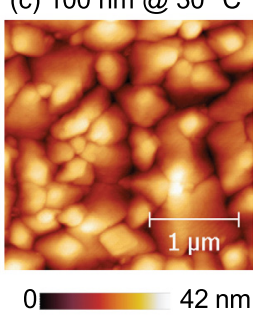

(e) $20 \mathrm{~nm} @ 0{ }^{\circ} \mathrm{C}$ and $30^{\circ} \mathrm{C}$ - profiles

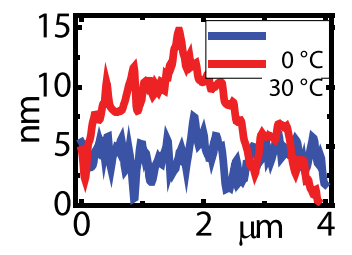

FIG. 2. (a) AFM morphology of the LSMO surface. (b) and (c) AFM micrographs showing the morphology of $3 \mathrm{~nm}$ and $100 \mathrm{~nm}$ thick pentacene films, respectively, deposited at $30^{\circ} \mathrm{C}$ on LSMO; (d) AFM micrograph of $20 \mathrm{~nm}$ thick pentacene film deposited at $0^{\circ} \mathrm{C}$ on LSMO; (e) peak-to-valley comparison of $20 \mathrm{~nm}$ films deposited at $0^{\circ} \mathrm{C}$ and $30^{\circ} \mathrm{C}$.

Figure 2(b) shows an AFM micrograph of a pentacene film with a nominal thickness of $3 \mathrm{~nm}$ deposited on LSMO at $30^{\circ} \mathrm{C}$. The first monolayer forms a wetting layer with a morphology which is radically different from the one of the LSMO. The subsequent molecules grow on this pentacene wetting layer and form three dimensional islands with an anisotropic shape, presumably due to the elongated shape of the pentacene molecules; anisotropic growth of pentacene on $\mathrm{SiO}_{2}$ is well known. ${ }^{22}$

Apart from the wetting layer, growth is anisotropic at all stages: Figure 2(c) shows the surface of a $100 \mathrm{~nm}$ nominal thickness pentacene film deposited at room temperature on LSMO. The islands in the $100 \mathrm{~nm}$ thick film are smaller and with a higher density than the islands at earlier stages of growth. This effect is the signature of a growth that is limited by the Ehrlich-Schwoebel (ES) barrier, which prevents the diffusing molecules from going over the edge of a terrace, thus confining them on a step. ${ }^{23}$ ES barrier-controlled growth leads to a three dimensional growth which results in a roughening of the film. ${ }^{24}$ This roughening is called "rapid" because it grows with the film thickness faster than in the random deposition model (RD), where molecules attach where they arrive, without diffusion. ${ }^{25}$ In general, it is valid that $R_{r m s} \sim t^{\beta}$, where $t$ is the nominal film thickness and $\beta$ is the growth exponent; in the RD model $\beta=0.5{ }^{25}$ Rapid roughening occurs when $\beta>0.5$, and is known for pentacene and other organic molecules deposited on $\mathrm{SiO}_{2},{ }^{25,26}$ for pentacene deposited on LSMO we obtain $\beta=0.6 \pm 0.1$.

Figure 2(d) reports the morphology of a $20 \mathrm{~nm}$ thick pentacene film deposited on LSMO at a measured temperature of $0{ }^{\circ} \mathrm{C}$. A higher nucleation rate is observed but not a higher roughness: $R_{r m s} \sim 1.3 \mathrm{~nm}$ and peak-to-valley roughness is about $10 \mathrm{~nm}$. Figure 2(e) compare the peak-to-valley roughness of two $20 \mathrm{~nm}$ thick films deposited at RT and $0{ }^{\circ} \mathrm{C}$ - the AFM morphology of $20 \mathrm{~nm}$ pentacene films deposited on LSMO is comprehensively described in Ref. 27. The rapid roughening seems to be suppressed and the pentacene film looks more compact: low temperature deposition 
could be a promising approach for devices with vertical transport.

More information comes from the scaling exponent $\alpha$ (static roughness exponent), obtained from the height-height correlation function $g: g_{\left(t_{x}, t_{y}\right)}=\frac{1}{N} \iint\left(z_{(x, y)}-z_{\left(x+t_{x}, y+t_{y}\right)}\right)^{2} d x d y$, where $z$ is the height of the point having $(x, y)$ coordinates and $N$ is the total number of points. For $t_{x}, t_{y} \ll \xi$, $g \sim R^{2 \alpha}$, where $\xi$ is the surface correlation length and $R=\sqrt{t_{x}{ }^{2}+t_{y}{ }^{2}} \cdot{ }^{26,28}$ For pentacene films grown on LSMO between $30^{\circ} \mathrm{C}$ and $60^{\circ} \mathrm{C}, \alpha$ does not depend on the substrate temperature but increases with the film thickness from $0.67 \pm 0.05$ at $3 \mathrm{~nm}$ to $0.80 \pm 0.03$ at $100 \mathrm{~nm}$. These values are indicative of a diffusion limited growth, ${ }^{29}$ a picture which will be assumed in what follows.

Figure 3 reports the topography of $3 \mathrm{~nm}$ thick pentacene films deposited at different temperatures. The size of the pentacene islands increases with deposition temperature, as does the interisland distance. Figure 3(f) shows that the interisland region in a sample deposited at $75^{\circ} \mathrm{C}$ is very similar to bare LSMO. While the LSMO surface looks completely covered when a $3 \mathrm{~nm}$ pentacene film is deposited at $30^{\circ} \mathrm{C}$, the coverage is not complete and the wetting layer disappears when pentacene is deposited above $40-45^{\circ} \mathrm{C}$. The thermal energy at $40-45^{\circ} \mathrm{C}$ is sufficient to overcome the interaction of pentacene molecules with the substrate, while on $\mathrm{SiO}_{2}$ a temperature of at least $80^{\circ} \mathrm{C}$ is needed. ${ }^{24}$ The flat morphology over a large area shown in Figures 3(b)-3(e) has been previously observed only on $\mathrm{SiO}_{2}$, and only when the growth was assisted by a supersonic gas flow that gave molecules a high kinetic energy and hence a high surface diffusivity. ${ }^{30}$ The analysis of the effective coverage, obtained by measuring the volume of the islands, ${ }^{15}$ shows that it decreases from $75 \%$ at a substrate temperature of $45{ }^{\circ} \mathrm{C}$ to $55 \%$ at $75^{\circ} \mathrm{C}$ : at this temperature desorption could start to play a significant role. ${ }^{31}$ All together, data in Figure 3 demonstrate that the molecule-substrate interaction is weak when pentacene grows on LSMO.

The energy landscape was studied quantitatively by means of the stable island density model, ${ }^{32}$ according to which the island surface density is given by $n \approx \exp \left(E_{N} / k_{\mathrm{B}} T\right)$, where $E_{N}$ is a global nucleation energy. When islands grow by capturing both the molecules landing on them directly and those that diffused from other sites on the substrate, $E_{N}=\frac{E_{i *}+i^{*} \cdot E_{D}}{\left(i^{*}+2\right)}$ or $\frac{E_{i *}^{*}+i^{*} \cdot E_{D}}{\left(i^{*}+2.5\right)}$ depending on whether the growth bi-dimensional or three-dimensional, respectively. ${ }^{32} i^{*}$ is the critical molecules number, i.e., the minimum number of molecules needed to form a stable nucleus, $E_{D}$ is the barrier to molecules diffusion on the substrate and $E_{i^{*}}$ is the formation energy for a stable nucleus. $E_{i^{*}}$ is expressed as $E_{i^{*}}=\sum_{m=2}^{i^{*}} E_{m}$, where $E_{m}$ is the formation energy of a nucleus of $m$ molecules.

In order to apply this nucleation model, we excluded the data from deposition at $30^{\circ} \mathrm{C}$ and at $75^{\circ} \mathrm{C}$; in the former case because of the wetting layer which looked to disappears at $\mathrm{T} \geq 40{ }^{\circ} \mathrm{C}$, in the latter because desorption started to play a significant role. From the Arrhenius plot in Figure 4(a), we extracted $E_{N}=0.65 \pm 0.05 \mathrm{eV}$ for $40 \leq \mathrm{T} \leq 60^{\circ} \mathrm{C}$. This energy scale is confirmed by ultra-violet photoemission spectroscopy measurements of a $0.4 \mathrm{eV}$ energy dipole between pentacene and $\mathrm{LSMO}^{27}$ which is low if compared to the $1 \mathrm{eV}$ found for pentacene deposited on Co. ${ }^{33,34}$ The $E_{N}$ value for pentacene on LSMO can be compared with the nucleation energy found for pentacene grown on $\mathrm{SiO}_{2}$ over the same temperature range: $E_{N}=0.78 \pm 0.05 \mathrm{eV}$ was found for a growth rate of $0.007 \mathrm{~nm} / \mathrm{s},{ }^{35}$ which is similar to the one used in the present experiment. At higher growth rates $(0.03 \mathrm{~nm} / \mathrm{s}) \quad \sum_{m=2}^{i^{*}} E_{m}+i^{*} E_{D}=1.13 \pm 0.04 \mathrm{eV}$ and $i^{*}=3$ were reported, ${ }^{36}$ hence $E_{N}=0.23 \pm 0.01 \mathrm{eV}$. Considering that $E_{N}$ varies with the growth rate, ${ }^{31}$ the value we extracted for pentacene on LSMO is compatible with the one for $\mathrm{SiO}_{2}$ substrates (Figure $4(\mathrm{~b})$ ), assuming that at lower rates $E_{N}$ levels off rather than diverging. ${ }^{35,37}$ Pentacene nucleation and growth on LSMO are much closer to those on $\mathrm{SiO}_{2}$ than those on $\mathrm{Co}^{34}$

The diffusion mechanism was analysed in order to disentangle the contributions of diffusion and nucleation in $E_{N}$. The surface diffusion length is defined as $x_{s}=\sqrt{D_{s} \tau}$, where $D_{s}$ is the diffusion coefficient and $\tau$ is the residence time, i.e., the time the molecule moves on the substrate before attaching or desorbing. $x_{s}$ is estimated as the mean distance between the centre of the islands. ${ }^{38}$ Since $D_{s}=a^{2} \omega e^{\left(E_{D} / k_{\mathrm{B}} T\right)}$ and $\tau=\omega^{-1} e^{\left(E_{a} / k_{\mathrm{B}} T\right)}$, where $a$ and $\omega$ are the pentacene molecules' jump distance and jump rate, respectively, the diffusion distance is $x_{s} \approx e^{\left(\left(E_{D}+E_{a}\right) / k_{\mathrm{B}} T\right)}$. Due to the above consideration about the diffusion limited character of the growth, we can approximate $x_{s}$ with $x_{s} \sim e^{\left(E_{D} / 2 k_{\mathrm{B}} T\right)}$, which gives $E_{D}=0.7 \pm 0.2 \mathrm{eV}$ for $\mathrm{T} \geq 40^{\circ} \mathrm{C}$ (Figure $4(\mathrm{c}$ )).

Based on the values of $E_{N}$ and $E_{D}$, we discuss $i^{*}$. If the smallest stable nucleus were a dimer, $E_{i^{*}=2} \sim 1.5 \pm 0.5 \mathrm{eV}$, in contrast with the calculated value of $0.67 \mathrm{eV} .{ }^{35}$ If we instead assume that the smallest stable nucleus is a trimer, $E_{i^{*}}=E_{2}+E_{3} \sim 1.5 \pm 0.6 \mathrm{eV}$. This seems reasonable and a similar trend in formation energies was reported for the benzene molecule, which comes from the same family to that of pentacene. ${ }^{39}$ Since $i^{*}=4$ would lead to $E_{i^{*}} \sim 1.4 \pm 0.5 \mathrm{eV}$, which seems too low, $i^{*}=3$ was most convincing estimate.
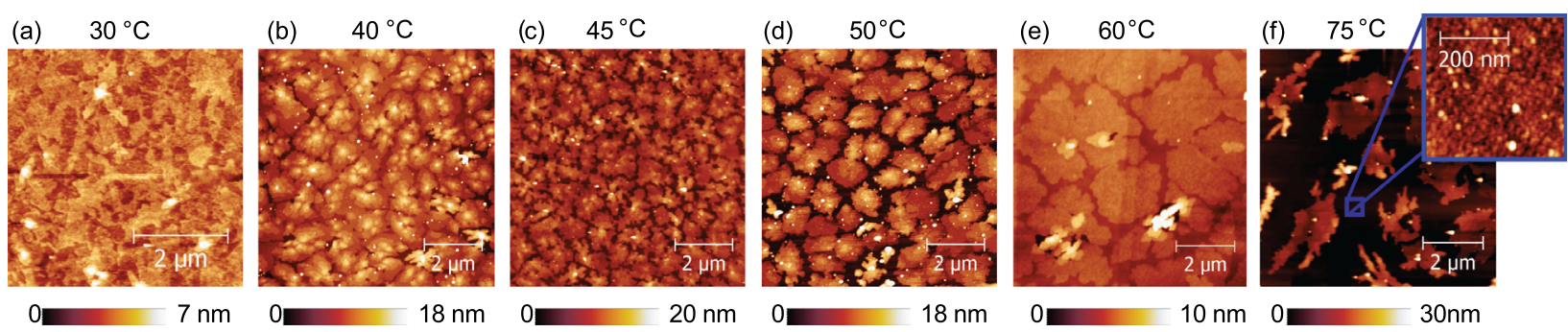

FIG. $3.3 \mathrm{~nm}$ thick pentacene films deposited at substrate temperatures from 30 to $75^{\circ} \mathrm{C}$ as indicated; the inset in (f) shows the interisland region with bare LSMO, $R_{r m s}=0.16 \mathrm{~nm}$, typical for LSMO. 

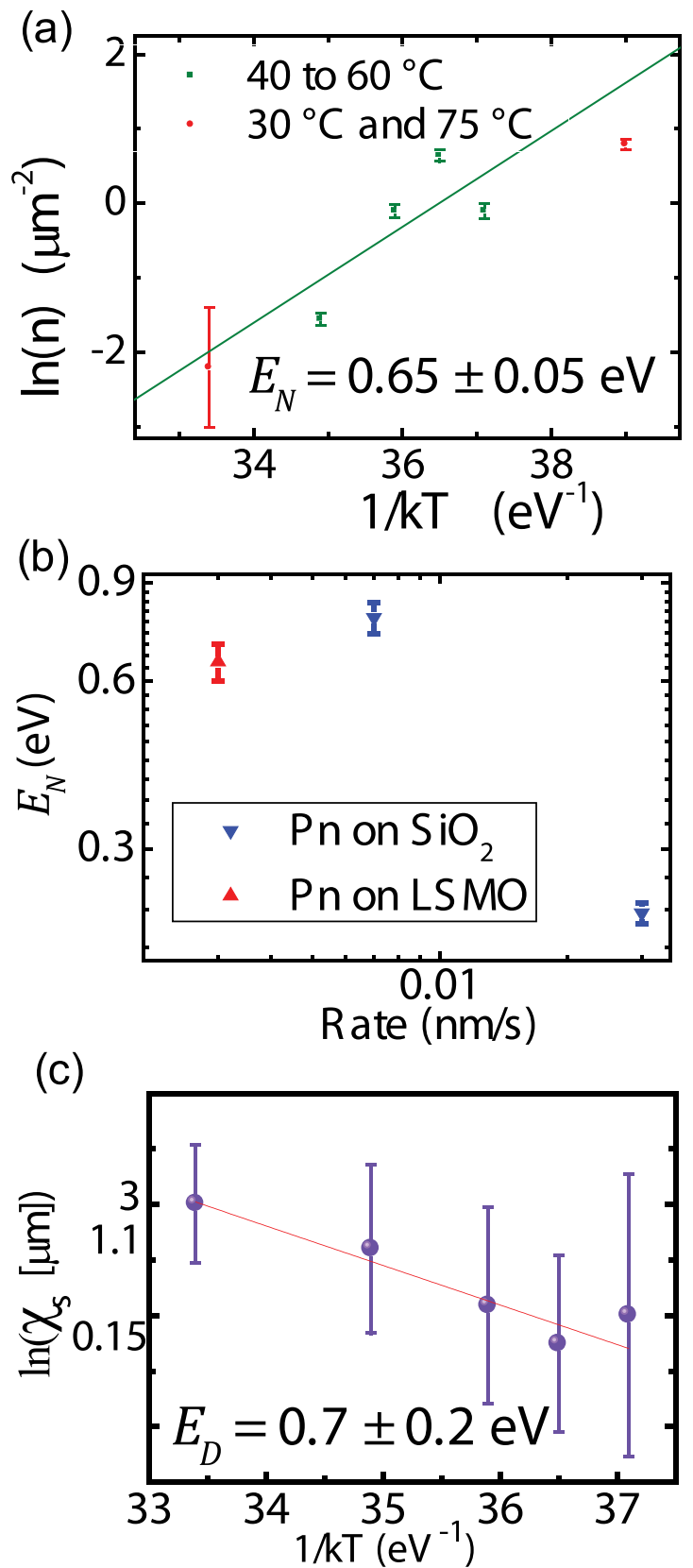

FIG. 4. (a) Islands density Arrhenius plot, from which we obtained $E_{N}$; the points from $40^{\circ} \mathrm{C}$ to $60^{\circ} \mathrm{C}$ are used (green square), while the red circles points are discarded because other mechanisms play a role; (b) rate dependence of $E_{N}$ for pentacene ( $\left.\mathrm{Pn}\right)$ on $\mathrm{SiO}_{2}$ and $\mathrm{LSMO}$, data for the $\mathrm{SiO}_{2}$ substrates come from literature $;^{35,36}$ (c) Arrhenius plot of the diffusion length, from which we obtained $E_{D}$.

In conclusion, we have shown that pentacene-based spin valves show spintronic effect up to room temperature.

Furthermore, we have shown that pentacene growth on LSMO is diffusion limited between $40^{\circ} \mathrm{C}$ and $60^{\circ} \mathrm{C}$; we extracted the nucleation energy and the diffusion barrier, finding a weak interaction picture. When deposited at $0{ }^{\circ} \mathrm{C}$, the surface showed lower peak to valley roughness. The observed morphology can strongly impact devices performance in ways that depend on whether the device is planar or vertical.

We firmly believe that this work not only demonstrate the feasibility of pentacene-based vertical spin valves showing that pentacene has great potential in organic spintronics applications but also maps a route towards the fabrication of improved pentacene-based spin valves.

The authors acknowledge the technical help from Federico Bona at CNR-ISMN in Bologna and the extensive use of the scanning probe microscopes at "Centro Interfacoltà Misure" of the University of Parma. Financial support from the FP7 Projects NMP-2010-SMALL-4263104 (HINTS), NMP3-SL-2010-246073 (GRENADA), and NMP3-LA-2010-246102 (IFOX) is acknowledged.

${ }^{1}$ M. Pope and C. E. Swenberg, Electronic Processes in Organic Crystals and Polymers (Oxford University Press, 1982).

${ }^{2}$ V. A. Dediu, L. E. Hueso, I. Bergenti, and C. Taliani, Nat. Mater. 8, 707 (2009).

${ }^{3}$ V. Dediu, M. Murgia, F. C. Matacotta, C. Taliani, and S. Barbanera, Solid State Commun. 122, 181 (2002).

${ }^{4}$ M. Prezioso, A. Riminucci, I. Bergenti, P. Graziosi, D. Brunel, and V. A. Dediu, Adv. Mater. 23, 1371 (2011); M. Prezioso, A. Riminucci, P. Graziosi, I. Bergenti, R. Rakshit, R. Cecchini, A. Vianelli, F. Borgatti, N. Haag, M. Willis, A. J. Drew, W. P. Gillin, and V. A. Dediu, ibid. 25, 534 (2013).

${ }^{5}$ V. A. Dediu and A. Riminucci, Nat. Nanotechnol. 8, 885 (2013).

${ }^{6}$ C. Wang, H. Dong, W. Hu, Y. Liu, and D. Zhu, Chem. Rev. 112, 2208 (2012).

${ }^{7}$ T. W. Kelley, L. D. Boardman, T. D. Dunbar, D. V. Muyres, M. J. Pellerite, and T. P. Smith, J. Phys. Chem. B 107, 5877 (2003).

${ }^{8}$ T. Ikegami, I. Kawayama, M. Tonouchi, S. Nakao, Y. Yamashita, and H. Tada, Appl. Phys. Lett. 92, 153304 (2008).

${ }^{9}$ S. Toshihiro, N. Hiroyuki, N. Takuya, F. Yutaka, Y. Yukio, H. Yasushi, H. Taro, and H. Tetsuya, Jpn. J. Appl. Phys., Part 147,1184 (2008).

${ }^{10} \mathrm{M}$. Popinciuc, Ph.D. thesis, University of Groningen, 2007.

${ }^{11}$ J.-Y. Hong, Y.-M. Chang, C.-H. Chuang, K.-S. Li, Y.-C. Jhang, H.-W. Shiu, C.-H. Chen, W.-C. Chiang, and M.-T. Lin, J. Phys. Chem. C 116, 21157 (2012).

${ }^{12}$ I. Bergenti, V. Dediu, M. Prezioso, and A. Riminucci, Philos. Trans. R. Soc., A 369, 3054 (2011).

${ }^{13}$ P. Graziosi, M. Prezioso, A. Gambardella, C. Kitts, R. K. Rakshit, A. Riminucci, I. Bergenti, F. Borgatti, C. Pernechele, M. Solzi, D. Pullini, D. Busquets-Mataix, and V. A. Dediu, Thin Solid Films 534, 83 (2013).

${ }^{14}$ M. P. de Jong, V. A. Dediu, C. Taliani, and W. R. Salaneck, J. Appl. Phys. 94, 7292 (2003).

${ }^{15}$ D. Nečas and P. Klapetek, Cent. Eur. J. Phys. 10, 181 (2012).

${ }^{16}$ V. Dediu, L. E. Hueso, I. Bergenti, A. Riminucci, F. Borgatti, P. Graziosi, C. Newby, F. Casoli, M. P. De Jong, C. Taliani, and Y. Zhan, Phys. Rev. B 78, 115203 (2008).

${ }^{17}$ T. S. Santos, J. S. Lee, P. Migdal, I. C. Lekshmi, B. Satpati, and J. S. Moodera, Phys. Rev. Lett. 98, 016601 (2007); D. Sun, L. Yin, C. Sun, H. Guo, Z. Gai, X. G. Zhang, T. Z. Ward, Z. Cheng, and J. Shen, Phys. Rev. Lett. 104, 236602 (2010).

${ }^{18}$ A. A. Sidorenko, C. Pernechele, P. Lupo, M. Ghidini, M. Solzi, R. De Renzi, I. Bergenti, P. Graziosi, V. Dediu, L. Hueso, and A. T. Hindmarch, Appl. Phys. Lett. 97, 162509 (2010); Y. Q. Zhan, X. J. Liu, E. Carlegrim, F. H. Li, I. Bergenti, P. Graziosi, V. Dediu, and M. Fahlman, Appl. Phys. Lett. 94, 053301 (2009).

${ }^{19}$ H. Chen, Z. Liu, L. Zhu, and K. Yao, "Giant Magnetoresistance Effects in the Organic spin valve $\mathrm{La}_{0.67} \mathrm{Sr}_{0.33} \mathrm{MnO}_{3} /$ Polyaniline/Co with Polyaniline as Spacer" (published online), available at http://www.paper.edu.cn/en_ releasepaper/content/4506953.

${ }^{20}$ C. Barraud, P. Seneor, R. Mattana, S. Fusil, K. Bouzehouane, C. Deranlot, P. Graziosi, L. Hueso, I. Bergenti, V. Dediu, F. Petroff, and A. Fert, Nat. Phys. 6, 615 (2010).

${ }^{21}$ J. M. De Teresa, A. Barthélémy, A. Fert, J. P. Contour, R. Lyonnet, F. Montaigne, P. Seneor, and A. Vaurès, Phys. Rev. Lett. 82, 4288 (1999).

${ }^{22}$ A. C. Mayer, R. Ruiz, R. L. Headrick, A. Kazimirov, and G. G. Malliaras, Org. Electron. 5, 257 (2004).

${ }^{23}$ M. G. Lagally and Z. Zhang, Nature 417, 907 (2002).

${ }^{24}$ A. Al-Mahboob, Y. Fujikawa, T. Sakurai, and J. T. Sadowski, Adv. Funct. Mater. 23, 2653 (2013).

${ }^{25}$ J. Krug, Physica A 340, 647 (2004).

${ }^{26}$ A. Brillante, I. Bilotti, R. G. Della Valle, E. Venuti, A. Girlando, M. Masino, F. Liscio, S. Milita, C. Albonetti, P. D’Angelo, A. Shehu, and F. Biscarini, Phys. Rev. B 85, 195308 (2012); A. C. Dürr, F. Schreiber, K. A. Ritley, V. Kruppa, J. Krug, H. Dosch, and B. Struth, Phys. Rev. Lett. 90, 016104 (2003). 
${ }^{27}$ F. Li, P. Graziosi, Q. Tang, Y. Zhan, X. Liu, V. Dediu, and M. Fahlman, Phys. Rev. B 81, 205415 (2010).

${ }^{28}$ F. Biscarini, P. Samorí, O. Greco, and R. Zamboni, Phys. Rev. Lett. 78, 2389 (1997).

${ }^{29}$ J. Villain, J. Phys. I 1, 19 (1991).

${ }^{30}$ T. Toccoli, A. Pallaoro, M. Tonezzer, N. Coppedè, and S. Iannotta, SolidState Electron. 52, 417 (2008); Y. Wu, T. Toccoli, J. Zhang, N. Koch, E. Iacob, A. Pallaoro, S. Iannotta, and P. Rudolf, Appl. Phys. A 95, 21 (2009).

${ }^{31}$ S. Pratontep, M. Brinkmann, F. Nüesch, and L. Zuppiroli, Phys. Rev. B 69, 165201 (2004).

${ }^{32}$ J. A. Venables, G. D. T. Spiller, and M. Hanbucken, Rep. Prog. Phys. 47, 399 (1984).
${ }^{33}$ M. Popinciuc, H. T. Jonkman, and B. J. van Wees, J. Appl. Phys. 100, 093714 (2006).

${ }^{34}$ M. V. Tiba, W. J. M. de Jonge, B. Koopmans, and H. T. Jonkman, J. Appl. Phys. 100, 093707 (2006).

${ }^{35}$ S. Pratontep, F. Nüesch, L. Zuppiroli, and M. Brinkmann, Phys. Rev. B 72, 085211 (2005).

${ }^{36}$ M. Tejima, K. Kita, K. Kyuno, and A. Toriumi, Appl. Phys. Lett. 85, 3746 (2004).

${ }^{37}$ J. G. Amar and F. Family, Phys. Rev. Lett. 74, 2066 (1995).

${ }^{38}$ B. Dam and B. Stauble-Pumpin, J. Mater. Sci.: Mater. Electron. 9, 217 (1998).

${ }^{39}$ H. Krause, B. Ernstberger, and H. J. Neusser, Chem. Phys. Lett. 184, 411 (1991). 